Ann. Zootech., I964, 13 (I), 87-92

\title{
APTITUDE DES TAUREAUX UTILISABLES EN INSÉMINATION ARTIFICIELLE A LA PRODUCTION DE SEMENCE CAPABLE DE SUPPORTER LA CONGÉLATION
}

\author{
M. GOFFAUX \\ Laboratoire de Physiologie de la Reproduction, \\ Centre national de Recherches zootechniques, Jouy-en-Josas (Seine-et-Oise); \\ Laboratoire de contrôle biologique et sanitaire des Reproducteurs, École nationale vétérinaire, Alfort
}

SOMMAIRE

I92 éjaculats de 48 taureaux ont été congelés dans un congélateur automatique à vapeurs d'azote après dilution dans un dilueur lait écrémé-glycérol et le pourcentage de spermatozoïdes mobiles apprécié au microscope avant et après congélation.

Les corrélations trouvées entre pourcentages de spermatozoïdes mobiles avant et après congélation $(0,48$ inter-éjaculats et 0,68 inter-taureaux. $P<0,01)$ permettent d'éliminer avant congélation les taureaux et les éjaculats particulièrement mauvais. Une méthode pratique de sélection en vue de la congélation est indiquée.

Dans le domaine de 1'Insémination Artificielle, de nombreuses recherches ont mis en évidence, entre taureaux, des différences qui s'expriment de façon immédiate par le taux de spermatozoïdes mobiles à l'examen microscopique de leurs éjaculats.

Alors que ces différences se manifestent régulièrement au cours des expérimentations portant sur la congélation, lorsque celles-ci comportent un nombre suffisant de taureaux non spécialement sélectionnés, chacun d'eux étant récolté plusieurs fois (BUCH, SMITH et TYLER, I956; O'DELI, et HURST, I956 ; ERIKsON et GRAHAM, I954; KENNELY I960) elles apparaissent beaucoup moins nettement lorsque le matériel animal est constitué par des taureaux dont le sperme, employé régulièrement par un Centre d'Insémination en vue de la congélation, porte la marque d'une sélection plus ou moins poussée des animaux et des éjaculats (BARTLETT et EliIOT, I960). Il reste à préciser l'importance de ces différences, observées après congélation, relativement à celles qui sont trouvées lors de 
l'emploi de sperme frais ainsi que leur liaison avec ces dernières. En particulier lorsque des écarts trop profonds imposent l'élimination de certains taureaux, la détermination de la proportion de tels animaux revêt un intérêt économique évident. D'après HERMANN (I96I), au moins $25 \mathrm{p}$. Ioo des taureaux pris au hasard seraient inadéquats aux techniques de congélation.

Devant l'absence, à notre connaissance, d'une démonstration réalisée sur une population de taureaux suffisamment grande, dont le seul critère de choix ait été leur utilisation en Insémination Artificielle, nous avons entrepris la présente étude.

\section{MATÉRIEL E'T TECHNIQUES}

48 taureaux (2I Frisons et 27 Normands) d'un âge moyen de 3 ans et demi, utilisés pendant 6 mois pour la réalisation de leurs inséminations de testage puis mis au repos, ont été récoltés 4 fois à environ 8 jours d'intervalle, chaque récolte comportant deux éjaculats obtenus sans excitation ni fausse monte préalable.

Le dilueur utilisé est analogue à celui expérimenté par ALMQUist en 1957.

La fraction non glycérolisée (Io p. Ioo de poudre de lait écrémé et $90 \mathrm{p}$. Ioo d'eau distillée en poids) est chauffée pendant to $\mathrm{mn}$ à $95^{\circ} \mathrm{C}$, et après refroidissement, reçoit 500 UI de pénicilline et $0,5 \mathrm{mg}$ de dihydrostreptomycine par $\mathrm{ml}$.

La fraction glycérolisée comprend en volume 80 p. 100 du milieu précédent et $20 \mathrm{p}$. I00 de glycérol, cette solution étant additionnée de $2,5 \mathrm{~g}$ de fructose monohydraté pour $100 \mathrm{ml}$.

Le dilueur final est formé du mélange à volume égal de chacune des deux fractions.

Le second éjaculat de chaque récolte a été prédilué, placé au réfrigérateur à $+4^{\circ} \mathrm{C}$ pendant 4 à 6 heures; puis sa concentration a été ajustée à $40.10^{6}$ spermatozoïdes par ml avant glycérolisation pour obtenir une concentration finale de $20.10^{6} \mathrm{par}$ ml, par l'addition du $\mathrm{r} / 3$ puis des $2 / 3 \mathrm{de}$ la solution glycérolisée à $30 \mathrm{mn}$ d'intervalle.

Trois heures après glycérolisation, la congélation du sperme ainsi dilué et conditionné en ampoules (2 supports de 4 ampoules par éjaculat) est effectuée dans un congélateur à vapeurs d'azote. Des différences importantes ayant été constatées avec cet appareil entre les courbes de descente de température enregistrées à partir d'ampoules occupant des positions différentes, le plan expérimental a été conçu de manière à contrôler la source possible de variation provenant de la position des ampoules dans l'appareil.

L'appréciation du taux de spermatozoïdes mobiles est faite I 5 heures après congélation à l'aide d'un microscope à contraste de phase grossissant $15^{\circ}$ fois, dont la platine est portée à une température voisine de $39^{\circ} \mathrm{C}$. Chaque lecture (faite dans l'ignorance de l'identité de l'échantillon examiné) aboutit à l'appréciation du taux de spermatozoïdes mobiles d'après les examens indépendants de deux ampoules par récolte.

\section{RÉSULTATS}

\section{Effets de la congélation}

Les résultats de l'étude de la population de taureaux d'après le critère du taux moyen de spermatozoïdes mobiles dans le sperme dilué avant et après congélation sont rassemblés dans le tableau I et les graphiques I et 2 .

La congélation exerce, sur les valeurs initiales du taux de spermatozoïdes mobiles, un effet multiplicatif non absolument rigoureux : Alors que le traitement multiplie en moyenne le pourcentage de spermatozoïdes mobiles par 0,46 (Graphique 2), les écarts-types inter-éjaculats et inter-taureaux sont multipliés par 0,65 (tableeu I). 
TABLEAU I

Caractéristiques statistiques (moyenne, écart-type et coefficient de variation) des mesures du taux de spermatozoides mobiles avant et après congélation

\begin{tabular}{c|c|c|c|c|c}
\hline \hline \multirow{2}{*}{ Traitement } & \multirow{2}{*}{ Moyenne } & \multicolumn{2}{|c|}{ Inter-Éjaculats } & \multicolumn{2}{|c}{ Inter-Taureaux } \\
\cline { 3 - 5 } & Écart-type & $\begin{array}{c}\text { Coefficient } \\
\text { variation }\end{array}$ & Écart-type & $\begin{array}{c}\text { Cofficient } \\
\text { variation }\end{array}$ \\
\hline Frais ................. & 62,2 & 15,5 & 0,25 & 10,6 & 0,17 \\
\hline Congelé ............... & 33,7 & 10,1 & 0,30 & 6,95 & 0,21 \\
\hline \hline
\end{tabular}

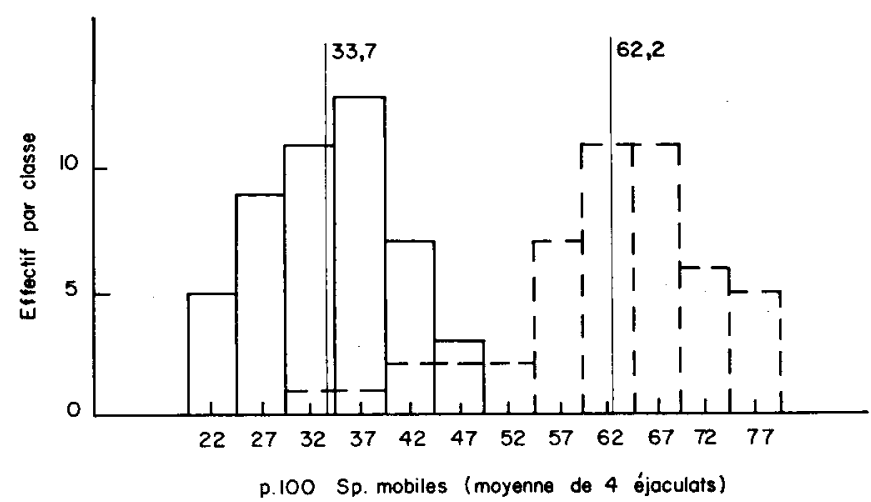

GRAPHIQUE I. Effectit de taureaux par classe de motilité moyenne observée sur sperme dilué. - — sperme frais

sperme congelé

\section{TABLEAU 2}

Corrélations, pour certains caractères de la motilité, entre sperme frais et sperme congelé, et régressions correspondantes

Mc : p. Ioo spermatozoïdes mobiles après congélation

Mf : p. Ioo spermatozoïdes mobiles avant congélation

Mi : motilité initiale notée de $\circ$ à 5 sur semence non diluée.

Nombre de données : pour les calculs concernant Mc et Mf, l'absence de I2 données correspond à la destruction intempestive de I2 ampoules après examen de Mf, sans qu'il ait été possible d'identifier les doses examinées.

\begin{tabular}{|c|c|c|c|c|}
\hline & & $\mathrm{Nb}$ données & Corrélations & Régression \\
\hline \multirow{2}{*}{ Mc et $\mathrm{Mf} *$} & Inter-éjaculats & 181 & $r=+0,48 \pm 0,06^{* *}$ & $\mathrm{Mc}=14+0,31 \mathrm{Mf} * *$ \\
\hline & Inter-taureaux & 48 & $r^{\prime}=+0,68 \pm 0,11 * *$ & $\mathrm{Mc}^{\prime}=5+0,46 \mathrm{Mf} * *$ \\
\hline \multirow[b]{2}{*}{ Mc et $\mathrm{Mi} *$} & Inter-éjaculats & 193 & $r=+0,44 \pm 0,06 * *$ & $\mathrm{Mc}=20+3,5 \mathrm{Mi} * *$ \\
\hline & Inter-taureaux & 48 & $r^{\prime}=+0,51 \pm 0,13^{* *}$ & $\mathrm{Mc}^{\prime}=20+3,6 \mathrm{Mi}^{* *}$ \\
\hline
\end{tabular}


En outre, la congélation rend homogènes les variances intra-taureaux qui apparaissent hétérogènes avant congélation (test de BAR'TLE'TT'T).

La liaison entre la motilité avant et après congélation pour chaque éjaculat et chaque taureau (moyenne de 4 éjaculats) est exprimée dans le tableau 2. Ia motilité de la semence fraîche a été appréciée d'une part avant dilution (motilité initiale), d'autre part environ Io $\mathrm{h} 30$ après dilution et séjour à $+4^{\circ} \mathrm{C}$ (taux de spermatozoïdes mobiles). Chacune des corrélations calculées est hautement significative, mais les valeurs de chaque coefficient ne diffèrent pas significativement entre elles.

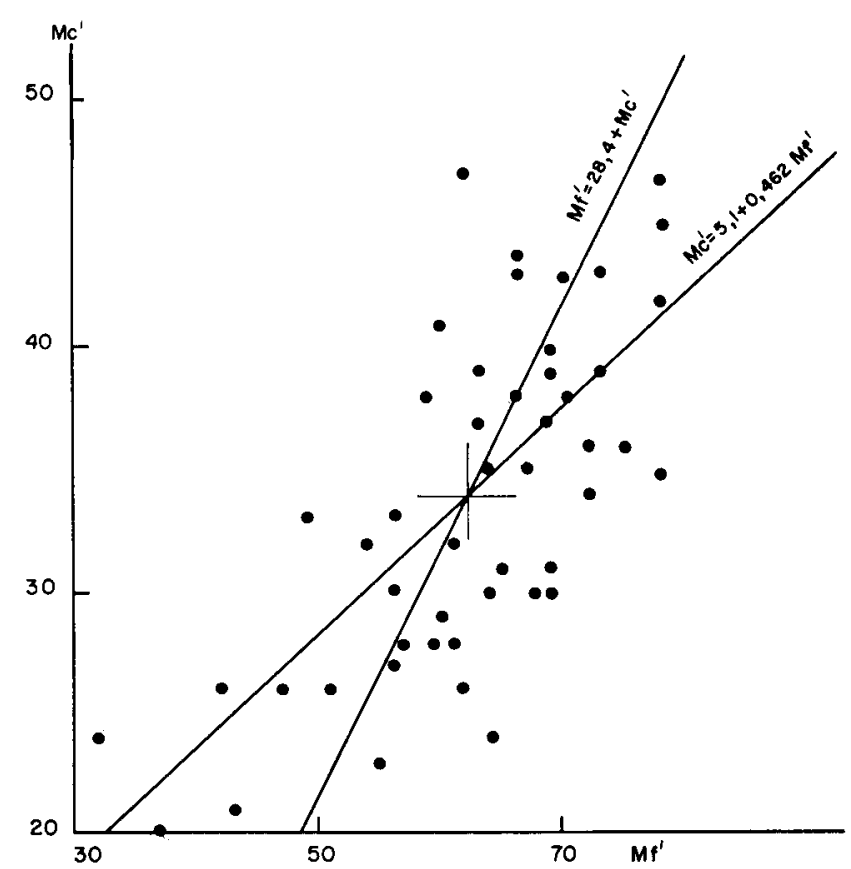

Graphique 2. Régression inter-taureaux, du taux de spermatozö̈des mobiles après congélation $\mathrm{Mc}^{\prime}$ sur le taux de spermatozoïdes mobiles avant congélation $\mathrm{Mf}^{\prime}$ et vice versa.

\section{DISCUSSION}

La liaison entre motilité avant et après congélation est certaine et s'exprime, lorsqu'on considère les moyennes par taureaux, par une corrélation assez étroite : $r=+0,68$.

Toutefois, les conclusions pratiques qu'on peut tirer de la corrélation indiquée sont limitées par le fait que 6 , parmi les 48 taureaux, ont un taux moyen de spermatozoïdes mobiles avant congélation inférieur à $50 \mathrm{p}$. IOO. Ils ne peuvent donc être considérés, d'après les critères habituels, comme "utilisables en Insémination Artificielle ». Si on limite la poptrlation étudiée aux 42 sujets " utilisables », 95 p. Ioo d'entre eux produisent une semence dont le taux moyen de spermatozoïdes mobiles après congélation est supérieur à 25 et 79 p. Ioo, une semence pour laquelle ce taux est au moins égal à 30 p. roo. 
Cependant la variabilité intra-taureaux est telle qu'une sélection limitée aux seuls taureaux entraîne l'obligation de détruire un nombre relativement élevé d'éjaculats congelés pour lesquels le taux de spermatozoïdes mobiles est inférieur à une valeur considérée comme la plus basse qui soit compatible avec leur utilisation en insémination. Aussi diverses tentatives fictives de sélection des éjaculats et (ou) des taureaux en vue de la congélation ont été essayées dans le but de réduire cette destruction onéreuse. La sélection nous a paru devoir porter à la fois :

- sur le taureau : elle entraîne l'élimination des animaux pour lesquels la movenne, calculée sur un nombre suffisant d'éjaculats, du taux de spermatozoïdes mobiles après congélation, est inférieure à une valeur limite ;

- sur l'éjaculat : elle se traduit par le rejet des échantillons où le taux de spermatozoïdes mobiles avant congélation est inférieur à une seconde valeur limite.

I)ans nos conditions expérimentales, une telle sélection n'est relativement efficace ( 7 p. Ioo seulement des éjaculats congelés devraient être détruits) sans être trop onéreuse $(95 \mathrm{p}$. Ioo des taureaux et $86 \mathrm{p}$. Ioo des éjaculats seraient aptes à être employés en congélation) que si la valeur limite acceptable du taux de spermatozoïdes mobiles après congélation n'est pas supérieure à $25 \mathrm{p}$. Ioo.

Ce rapport limite de trois spermatozoïdes immobiles pour un mobile semble d'ailleurs assorti d'une certaine marge de sûreté : rappelons que PickEтT (I96r-3) utilise toute semence congelée pour laquelle le taux de spermatozoïdes mobiles est supérieur à I7 p. Ioo, pourvu que chaque dose contienne au moins Io millions de spermatozoïdes mobiles.

D'ailleurs une expérimentation différente nous indiquera si l'emploi de semence ayant un taux de spermatozoïdes mobiles supérieur ou égal à 20 p. Ioo est compatible avec la sauvegarde d'une fécondance suffisante et dans quelles conditions.

Reçu pour publication en décembre ${ }^{9} \sigma_{3}$.

\section{REMERCIEMENTS}

Ce travail a pu être effectué grâce à un fonds de concours de l'U. N. C. E. I. A. et à l'aimable collaboration de l'Administration et du Personnel de la Coopérative d'Élevage et d'Insémination de Centre-Nord (Charmoy) auxquels je tiens à exprimer mes plus vifs remerciements.

\section{SUMMARY}

THE APTITUDE OF BULLS SUITABLE FOR USE IN ARTIFICIAL INSEMINATION FOR THE PRODUCTION OF SEMEN CAPABLE OF WITHSTANDING FREEZING

Four ejaculates of each of 48 bulls were collected, diluted in a skimmed milk-fructose-glycerolantibiotics medium, as described by ALMQUisT (1957), and frozen in a nitrogen vapour automatic refrigerator.

The study of the population of the ejaculates according to their motilities (percentage motile spermatozoa) examined before and after freezing led to the following conclusions:

I) the correlations between percentage motile spermatozoa before and after freezing were +0.48 between ejaculates, and +0.68 between bulls $(\mathrm{P}<0.0 \mathrm{I})$; 
2) various imaginary tests of selecting ejaculates and/or bulls have been carried out with respect to freezing.

After prior selection of 42 bulls whose ejaculates before freezing had an average motility greater than $5^{\circ}$, it was found that $95 \mathrm{p} .100$ of the bulls available in current Artificial Insemination produced a semen whose average percentage of motile spermatozoa after freezing was greater than 25 , and that $79 \mathrm{p}$. Ioo of them produced a semen whose average percentage of motile spermatozoa after freezing was greater than, or equal to, 3 .

\section{RÉFÉRENCES BIBLIOGRAPHIQUES}

Amman R. P., Almquist J. O., 1957. Freezing of bovine semen II. Effect of milk solids level, glycerol level, and fructose on freezability of bull spermatozoa in reconstituted and fresh skimmilk diluents. $J$. Dairy Sci., 40, I $542-1549$.

Bartlett D. E., ElliotT F. I., I960. The constancy of fertility of normal bulls as expressed in artificial insemination. Internat. J. Fertil., 5, 307.

Bratton R. W., Flood J. C., Foote R. H., Wearden S., Dunn H. O., ig57. Fertility of bovine spermatozoa stored at minus $79^{\circ} \mathrm{C}$ for one week and seventeen weeks. J. Dairy Sci., 40, I54-162.

Buch N. C., Smith V. R., Tyler W. J., I956. Bull and line differences in the survival of spermatozoa after freezing and thawing. J. Dairy Sci., 39, I 7 I 2-I 716 .

ERICKSON W. E., Graham E. F., 1959. Factors affecting the fertility of frozen bovine spermatozoa. $J$. Dairy Sci., 42, 520-528.

Graham E. F., Erickson W. E., Bayley N. D., I959. Effect of glycerol equilibration on frozen bovine spermatozoa. J. Dairy Sci., 40, 510-515.

Hermann H. A., i96r. Broad scale use of frosen semen. Proc. IV th Internat. Cong. anim. Reprod. The Hague, 5 th-gth June r96r. IV, 969-97 I.

Kennely J. J., Hoyt R. S., Foote R. H., Bratton R. W., I960. Survival rates of rapidly frozen bovine spermatozoa. J. Dairy Sci., 43, I I 40-I I 46.

O'Delt. W. T., Almquist J. O., 1957. Freezing bovine semen. I. Techniques for freezing bovine spermatozoa in milk diluents. J. Dairy Sci., 40, I 534-1 541.

O'DeLL G., HuRst V., 1956. The effect of glycerol equilibration time on the freezing of the bovine spermatozoa in egg-yolk sodium citrate and skimmilk semen extenders. J. Dairy Sci., 39, I I56-г г60.

Picket et al., I96r. Effects of $-196^{\circ} \mathrm{C}$ and $-79^{\circ} \mathrm{C}$ storage on the resistance of bull spermatozoa to three repeated freeze-thaw treatments. J. Dairy Sci., 44, 715-720.

Picketr et al., I96r. Correlation between laboratory stress tests and fertility of frozen bovine spermatozoa. J. Dairy Sci., 44, Ir34-I I40.

Picket et al., I96r. Preservation of bovine spermatozoa at $-79^{\circ} \mathrm{C}$ and $-196^{\circ} \mathrm{C}$. J. Dairy Sci., 44, $2089-2096$ 